\title{
Searching for signatures of stochastic excitation in stellar pulsations: a look at $\gamma$ Doradus stars ${ }^{\star}$
}

\author{
T. M. D. Pereira ${ }^{1, \star \star}$, J. C. Suárez ${ }^{2,3, \star \star \star}$, I. Lopes ${ }^{1,4}$, S. Martín-Ruiz ${ }^{2}$, P. J. Amado ${ }^{5,2}$, R. Garrido ${ }^{2}$, E. Rodríguez ${ }^{2}$, \\ V. Costa ${ }^{2}$, A. Rolland ${ }^{2}$, A. Arellano Ferro ${ }^{6}$, and J.-P. Sareyan ${ }^{7}$ \\ ${ }^{1}$ Centro Multidisciplinar de Astrofísica, Instituto Superior Técnico, Av. Rovisco Pais, 1049-001 Lisboa, Portugal \\ e-mail: tiago@mso.anu.edu.au \\ 2 Instituto de Astrofísica de Andalucía (CSIC), Apdo. 3004, 18080 Granada, Spain \\ 3 LESIA, Observatoire de Paris-Meudon, UMR 8109, France \\ 4 Centro de Geofísica de Évora, Departamento de Física, Évora, Portugal \\ 5 Univ. de Granada, Dpto. Física Teórica y del Cosmos, Campus Fuentenueva, Granada, Spain \\ ${ }^{6}$ Instituto de Astronomía, Universidad Nacional Autónoma de Mexico, Apdo. Postal 70-264, 04510 Mexico DF, Mexico \\ 7 GEMINI, Observatoire de la Côte d'Azur, UMR 6203, BP 4229, 06304 Nice Cedex 4, France
}

Received 4 April 2006 / Accepted 30 November 2006

\section{ABSTRACT}

\begin{abstract}
In stellar oscillations, the temporal variation in the amplitude of a given mode can yield some information about the interior of the star. In what concerns the excitation mechanism, it has been shown that for oscillations that are excited and damped by a physical process in stochastic equilibrium, the ratio of the standard deviation of the amplitude $\sigma_{\mathrm{A}}$ over the amplitude mean value $\mu_{\mathrm{A}}$ is of the order of 0.52 . This "statistical signature" is a general property of stochastically excited oscillations, so it can be used with any type of star - making it a powerful tool.

Although the method is simple, its reliability and robustness have yet to be proven. With the help of simulations, these points are addressed in the present work, and the observational requirements for this method to work are more clearly defined. We show that a number of amplitude measurements of about $10^{2}$ are required to get a good estimate of $\sigma_{\mathrm{A}} / \mu_{\mathrm{A}}$. The method is also tested against the complication of having a time series crowded with many modes (unresolvable in short time series), and results show that the theoretical relation of $\sigma_{\mathrm{A}} / \mu_{\mathrm{A}}$ still holds true.

Some of the motivation behind the new tests of the method involves applying it to $\gamma$ Doradus stars. The open question of what drives oscillations in these stars makes this statistical method very interesting when applied to $\gamma$ Doradus stars. A test of the method is performed using $\gamma$ Doradus observations. As expected, the results are inconclusive and demonstrate how ground-based observations of $\gamma$ Doradus stars hardly fulfill the applicability requirements of the method. We show, however, how a possible scenario of stochastic excitation in these stars could be detected with the COROT space mission.
\end{abstract}

Key words. methods: statistical - stars: statistics - stars: oscillations - stars: fundamental parameters

\section{Introduction}

The $\gamma$ Doradus pulsating stars are typically early F-type stars with luminosity class $\mathrm{V}$ or IV, displaying low visual amplitudes of a few hundredths of magnitude whose periods range from $0.5^{\mathrm{d}}$ to $3.0^{\mathrm{d}}$. Some $\gamma$ Doradus stars coexist with the $\delta$ Scuti variables inside the instability strip, but the majority are located on and outside the cool border. Close to the $\delta$ Scuti stars in the HR diagram, the $\gamma$ Doradus stars are representative of intermediatemass stars. Kaye et al. (1999) classified them as multi-periodic non-radial gravity mode oscillators, with low-degree and highradial order. An excellent work summarizing the evolution of the research in the field stars can be found in Zerbi \& Kaye (2002).

The majority of $\gamma$ Doradus stars have been discovered by accident. The relatively long periods and very low amplitudes make any observational detection very difficult. Some were

* Based on observations obtained at Sierra Nevada Observatory ("Instituto de Astrofísica de Andalucía", CSIC), Granada, Spain.

* Present address: RSAA, Mt. Stromlo Observatory, Cotter Rd., Weston, ACT 2611, Australia.

$\star \star \star$ Associate researcher at institute (3). detected while being used as comparison stars for differential photometry in multi-site campaigns of previously known $\delta$ Scuti variables. Since the list of 13 bona-fide $\gamma$ Doradus stars published by Kaye et al. (1999), a big effort has been made to find new members of the class. Important systematic searching for $\gamma$ Doradus star candidates were performed in different databases such as the Hipparcos Catalogue (ESA 1997) (Aerts et al. 1998; Handler 1999) and Geneva photometric database (Eyer \& Aerts 2000). In addition to this, systematic observations in open clusters have significantly contributed to increasing the number of possible $\gamma$ Doradus candidates (see Martín \& Rodríguez 2000; Kim et al. 2001; Choo et al. 2003; Martín 2003; Arentoft et al. 2005). In order to confirm their pulsational nature, many of these new candidates have been revisited (see Martín et al. 2003; Henry \& Fekel 2003; Henry et al. 2005, and references therein). In the Henry et al. (2005), an updated list of the 54 confirmed $\gamma$ Doradus stars is reported.

Special mention should be made of the earliest works attempting mode identification like, to name a few, Balona et al. (1996) who apply the line profile variations technique to $\gamma$ Doradus stars, Aerts \& Krisciunas (1996) who study the 
mode identification in the slowly pulsating F0V star 9 Aur, Zerbi et al. (1999) who reported the results of a multisite campaign on HR 8899, or Aerts \& Kaye (2001) who made a spectroscopic analysis of HD 207223.

Since the first theoretical instability of $\gamma$ Doradus stars proposed by Warner et al. (2003), significant improvements have been recently achieved for these stars in the framework of asteroseismology. These improvements concern the identification and the driving mechanism of their pulsation modes. To name a few (the more recent), for the mode identification, Moya et al. (2005) and Suárez et al. (2005) propose the frequency ratio method (FRM), which simultaneously constrains the mode identification and restricts the possible representative models. Furthermore, the robustness of the method for moderately rotating $\gamma$ Doradus stars is also shown (Suárez et al. 2005).

The driving mechanism of the $\gamma$ Doradus gravity modes has been a matter of debate during the past decade. The theory developed by Guzik et al. (2000) ascribes the origin of pulsation to convection. In particular, pulsations are considered as being driven by a periodic flux blocking at the base of the convective envelope. The balance between this flux-blocking driving and the radiative damping in the $g$-mode cavity explains the location of their instability strip. The authors used a frozen convection (FC) treatment in their non-adiabatic modelling. This approximation holds true only in a very thin region near the bottom of the convective zone, where the lifetime of the convective elements is longer than the pulsation periods. However, this is not the case in the rest of the convective zone. This problem has been recently solved by the time-dependent convection theory developed by Gabriel (1996) and Grigahcène et al. (2005). The new TDC models confirm the predictions proposed by Guzik et al. (2000), i.e., the periodic flux blocking at the base of the convective envelope as responsible for the driving of $g$ modes. Moreover, the balance between this flux and the radiative damping in the $g$-mode cavity explains the location of the instability strip (Dupret et al. 2004, 2005) for these stars.

The aim of this work is to identify in which conditions the signature of stochastic excitation can be detected in $\gamma$ Doradus stars, using the statistical method proposed by Pereira \& Lopes (2005), which relies on numerical simulations of stochastic excitation with some improvements that are important to the specific case of $\gamma$ Doradus stars. Fundamental observational problems inmediately arise when applying the statistical method to these stars: the period and amplitude stability over a given duration, which is necessary for the theory to be applied, and the time resolution to detect stochastic modes. Up to now, ground-based observations hardly fulfil such conditions. Aware of this, we illustrate the application of the method (its problems and results) to ground-based $\gamma$ Doradus star observations using the $\gamma$ Doradus star HD 22702, and we dicuss the results as a starting point for future statistical analysis of space obsevartions.

The outline of this paper is as follows. In the next section a brief description of the method used to identify stochastically excited oscillations is given. In Sect. 3 we use simulations of stochastic oscillators to test the amplitude distribution and the effect of a crowded spectrum on the amplitude distribution. In Sect. 4 we turn our attention to the applicability of the method by testing how many values of amplitude (and how they are sampled) are required for the statistical method to yield precise results. In Sect. 5 we discuss the application of the method to $\gamma$ Doradus stars, we make a test with ground-based observations, and then see how data from COROT might help. Finally, we draw our conclusions in Sect. 6.

\section{Identifying stochastically excited oscillations}

\subsection{The method}

Based on the statistical properties of stochastically excited oscillators, a simple diagnostic method can be established to probe the oscillations driving mechanism (see Pereira \& Lopes 2005). We consider a single damped harmonic oscillator, whose amplitude is randomly excited. If one can measure the oscillator's energy over time intervals much smaller than the damping time, then the energy distribution naturally follows a Boltzmann distribution (see Kumar et al. 1988):

$p(E)=\frac{1}{\langle E\rangle} \exp \left(-\frac{E}{\langle E\rangle}\right)$,

where $\langle E\rangle$ is the mean energy. To obtain the amplitude distribution, one has in mind that

$p(E) \mathrm{d} E=p(A) \mathrm{d} A$

and that for an oscillator $E$ is proportional to $A^{2}$ :

$E=\frac{1}{2} k A^{2}$

Using (1)-(3) one obtains a Rayleigh-type distribution for the amplitude distribution:

$p(A)=\frac{2 A}{\left\langle A^{2}\right\rangle} \exp \left(-\frac{A^{2}}{\left\langle A^{2}\right\rangle}\right)$,

where $\left\langle A^{2}\right\rangle$ is a parameter connected with the mode energy. This distribution has an interesting relationship between its mean $\langle A\rangle$ and its standard deviation $\sigma(A)$ :

$\sigma(A)=\left(\frac{4}{\pi}-1\right)^{1 / 2}\langle A\rangle \approx 0.52\langle A\rangle$.

This simple relation holds true when an oscillator is in stochastic equilibrium (Lopes 2006, in preparation). By computing the statistic $\sigma(A) /\langle A\rangle$, one can probe for the existence of stochastic excitation in a given oscillator. This method is easily applied to stellar oscillations, provided that one is able to observe and identify a given mode over a set of nights.

One useful tool when probing the excitation mechanism in a star is the so-called excitation diagram, a $\sigma(A)$ vs. $\langle A\rangle$ plot. The diagram is split in two regions, divided by the $y \approx 0.52 x$ line, which defines the stochastic equilibrium. The stochastically excited stellar oscillations are expected to be found around the 0.52 line, which is the most interesting zone of the diagram. The upper and lower regions of the diagram are believed to represent oscillations that are not in equilibrium. While most of the stellar oscillations that are not stochastically excited are believed to lie in the lower region $(\sigma(A)<0.52\langle A\rangle)$ of the diagram (some observational results are given in Pereira \& Lopes 2005), the upper region corresponds to non-equilibrium stochastic oscillations that have yet to be observed.

In Pereira \& Lopes (2005) a numerical study was made to investigate the limitations of this method, in the framework of a case study. We have refined this numerical study in order to make more general conclusions and to include some ingredients relevant to the study of $\gamma$ Doradus stars. 


\subsection{Numerical study and previous work}

The previous numerical study was made in order to answer the question: given that $n$ measurements of a mode's amplitude are made, what is the probability that $\sigma(A) /\langle A\rangle=0.52$ if the mode is stochastically excited? This approach led to the construction of the distributions of the sample $\sigma(A) /\langle A\rangle$, the tools used to set bounds on the expected $\sigma(A) /\langle A\rangle$. The bounds set on $\sigma(A) /\langle A\rangle$ depended naturally on the significance of this statistic. Although the factors affecting the significance of the statistic were not deeply analyzed, the previous results provided a basic answer to the initial question.

In the strategy of using $\sigma(A) /\langle A\rangle$ to detect stochastic excitation, there are two basic issues at stake: a physical process (what is the amplitude distribution?) and a statistical sampling process (if the oscillator is stochastic, what is the sample $\sigma(A) /\langle A\rangle$, given that we make $n$ measurements of $A$ ?). The previous approach entangled the two issues, making it harder to study each issue individually, therefore an improved numerical study was performed and the issues of the amplitude distribution (3) and the sampling (4) have been studied independently.

\section{Testing for the amplitude distribution}

In purely theoretical terms of a single-mode oscillation, the amplitude distribution is well defined by Eq. (4). This result can be confirmed with a simple simulation.

First, a large time series is generated, whose signal is generated as a stochastically excited damped harmonic oscillator. The signal of the stochastic oscillator can be constructed in a similar way as in Pereira \& Lopes (2005)

$S(t)=A_{1} \sin (\omega t)+A_{2} \cos (\omega t)$,

where, at each sample time $\Delta t$, the $A_{i}$ are reset according to the expression

$A_{i}(t+\Delta t)=\exp (-\eta \Delta t) A_{i}(t)+\mathcal{N}(0, \xi) \delta$,

where $\eta$ is the inverse of the damping time, and $\mathcal{N}(0, \xi)$ a random number drawn from a normal distribution with zero mean and $\xi$ variance, and $\delta$ is the average excitation factor.

Second, the time series is divided into multiple sections, "slices", and the amplitude is determined in each slice. Since each slice yields one amplitude value, by combining all the data we have a set of amplitude values, which we refer to as "population". To infer something about the amplitude distribution, we would simply compute a histogram of the population.

In the simple case of an oscillator with one frequency, we generated a signal with the following parameters: $v=1 \mathrm{mHz}$; damping time of 2.5 days; $\xi=0.1$; sampling time of $100 \mathrm{~s}$; total time span of 95 years (approx. $3 \times 10^{7}$ points) split in $10^{6}$ subsets (each with approximately $50 \mathrm{~min}$, or three periods). With the resulting amplitude population, a histogram was computed, and the result is given in Fig. 1. From the figure one can see that, as expected, the theoretical expression is a very good approximation.

Having confirmed the amplitude distribution, we can now go one step forward to discuss an important issue when trying to apply the method to $\gamma$ Doradus stars. Models of these stars (Moya et al. 2005; Suárez et al. 2005) show that they typically have several long (usually many hours or a few days), closelyspaced periods. These periods cause a complex beating effect, on an even longer time scale than the periods. In terms of groundbased observations, this presents two problems: (a) the long periods mean that one has to make several observations during a

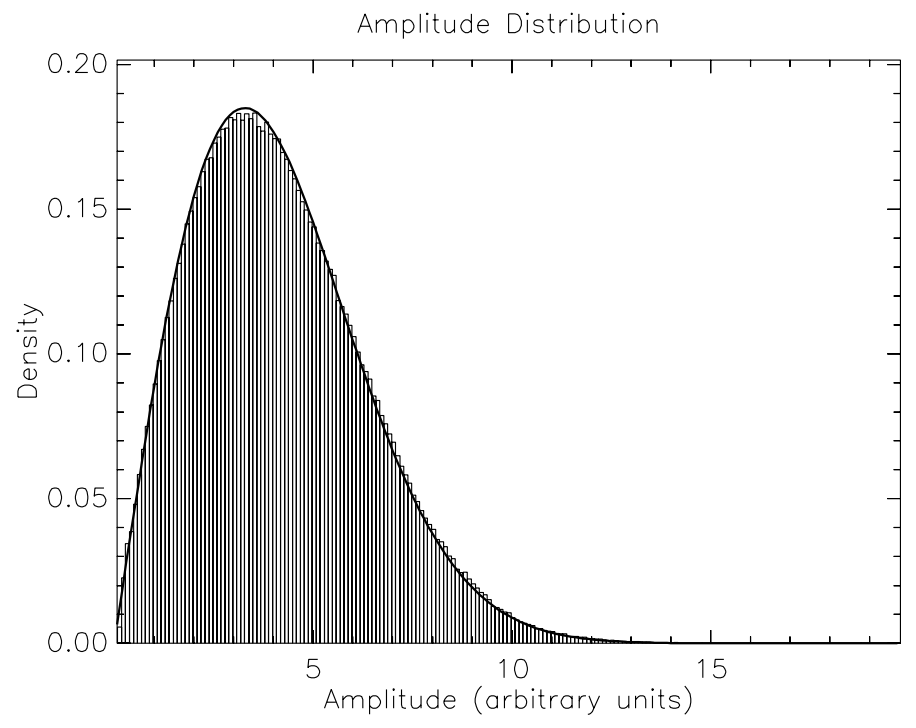

Fig. 1. Normalized amplitude histogram, based on the amplitude values determined in our simulation. The histogram comprises $10^{6}$ points. The solid line represents the amplitude distribution function from Eq. (4), with a chosen $\left\langle A^{2}\right\rangle$.

few nights in order to determine the amplitude of a mode and (b) the resolution needed to resolve the closely-spaced periods is very high, and very difficult to attain even with observations on several nights. At this stage we address point (b).

In a practical scenario, the closely-spaced modes typical of a $\gamma$ Doradus star mean that each amplitude measurement (even if only one mean value is determined in a set of nights) will be affected by a beating effect. Thus, an important question to ask when using the statistical method in these stars is what happens to the amplitude distribution in this scenario. Accordingly, we modified our simulation, accounting for this scenario. Instead of just one frequency, the generated signal was a sum of several frequencies. Originally at $v_{0}=1 \mathrm{mHz}$, the new frequencies began at $v_{0}$ and were incremented by $10 \mu \mathrm{Hz}^{1}$. This small separation yielded a beating effect whose period was around $27 \mathrm{~h}$, which was unresolvable in each slice of $50 \mathrm{~min}$. By keeping $\xi$ the same in each mode, the mean amplitudes were equal. In this way, our simulation created an amplitude population in which each amplitude value was somehow influenced by the beating effect (i.e., the values were multiplied by a circular function).

Some results from simulations with more than one mode can be seen in Fig. 2. It is clear from the figure that the width of the distribution, proportional to $\left\langle A^{2}\right\rangle$ increases with the number of modes. The $\left\langle A^{2}\right\rangle$, in turn, is proportional to $\langle E\rangle$, the mean mode energy. Adjusting the parameter in Eq. (4) to the different histograms, we found that $\left\langle A^{2}\right\rangle \approx k\left\langle A^{2}\right\rangle_{0}$, where $k$ is the number

1 It should be noted that the chosen values for the frequencies, frequency increments, damping time, sampling time, etc. are not very important per se (in fact, these imply much shorter periods than in $\gamma$ Doradus stars, for instance) but only in their relative values. The frequency increments were chosen so that they are 0.01 of the main frequency (which is consistent with the $\gamma$ Doradus case); the sampling time was chosen so that the resolution was good; the damping time was chosen so that it was much longer than the sampling time (condition for a stable stochastic excitation); the frequency was chosen so that at least three periods were present in each amplitude determination (for the given sample time) and so that in the end one would have around $10^{6}$ amplitude values in about $100 \mathrm{yr}$. The specific values do not influence the end result at all (only the relation between them), making it true to any stochastically excited oscillation. 


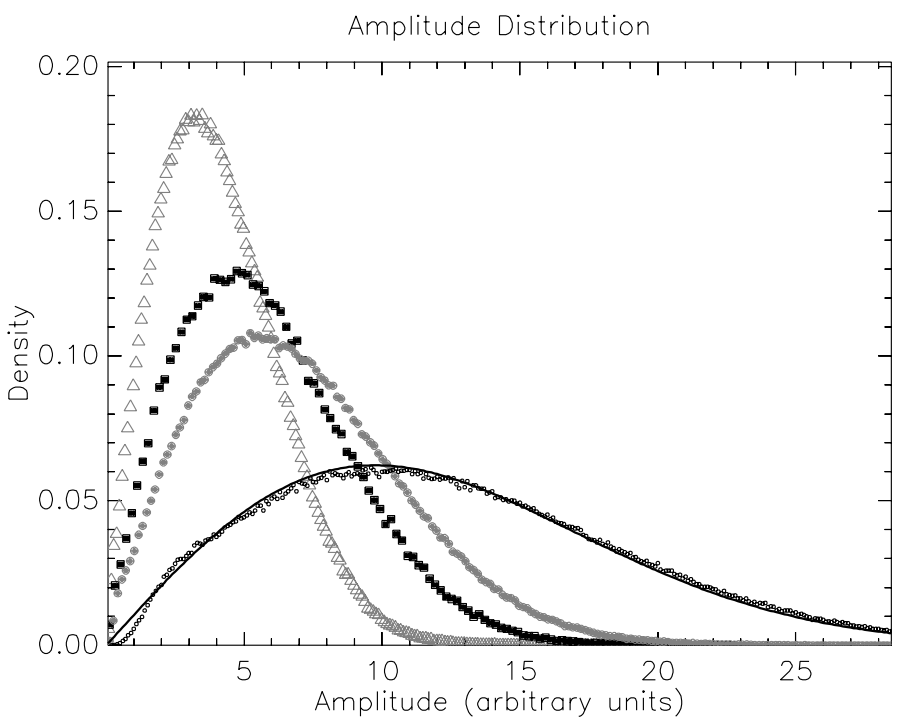

Fig. 2. Normalized amplitude histograms, based on the amplitude values determined in four simulations. Each histogram comprises $10^{6}$ points. The histograms correspond to, respectively from the higher to the lower peaks, one mode (gray triangles), two modes (black squares), three modes (gray filled circles), and 10 modes (small black open circles). For the histogram corresponding to 10 modes, a solid line has been plotted, representing the amplitude distribution function from Eq. (4), with a chosen $\left\langle A^{2}\right\rangle$.

of modes and $\left\langle A^{2}\right\rangle_{0}$ the parameter for the simulation with just one mode. As all the modes in the simulations had the same mean energy, this result indicated that overall the mean energies of each mode were being summed effectively. This means that, when unable to resolve the closely spaced frequencies, a set of amplitude measurements will yield a distribution whose $\left\langle A^{2}\right\rangle$ is the sum of the contributions of $\left\langle A^{2}\right\rangle_{i}$ of each unresolved mode.

As a side note, it is worth mentioning that the distributions from the simulations can slightly deviate from Eq. (4). Although the agreement is generally very good, we found a slight deviation from the Rayleigh distribution in the zone where the function started to rise, just before the peak. For instance, this effect can be seen, with a little effort, in Fig. 2, in the darkest histogram. This is, nevertheless, a minor detail that did not interfere with the statistic paramount to this work, $\sigma(A) /\langle A\rangle$.

\section{Testing for sampling effects}

The statistical method outlined in Sect. 2.1 tells us that for a stochastic oscillator, $\sigma(A) /\langle A\rangle$ should be around 0.52 . But when observing, only a limited set of values (measurements) of $A$ is available. This limited number of values will determine if the sample $\langle A\rangle$ and $\sigma(A)$ are good estimators of the true values. An important question should therefore be asked: how many amplitude measurements $n$ does one need to make a good measurement of $\sigma(A) /\langle A\rangle$ ? In other words: given that one measures $n$ values of $A$, how does that affect the sample $\sigma(A) /\langle A\rangle$ ? It turns out that not only is the number of measurements $n$ important for correctly determining the $\sigma(A) /\langle A\rangle$ but also how these measurements are made (sampling type), as explained in the next paragraphs.

Suppose that we have a large population of amplitude values, each one determined in contiguous sets of a large time series. To simulate amplitude measurements (i.e., extracting $A$ from observational time series), we pick $n$ amplitude values from our population. Then the sample $\sigma(A)$ and $\langle A\rangle$ are computed from the

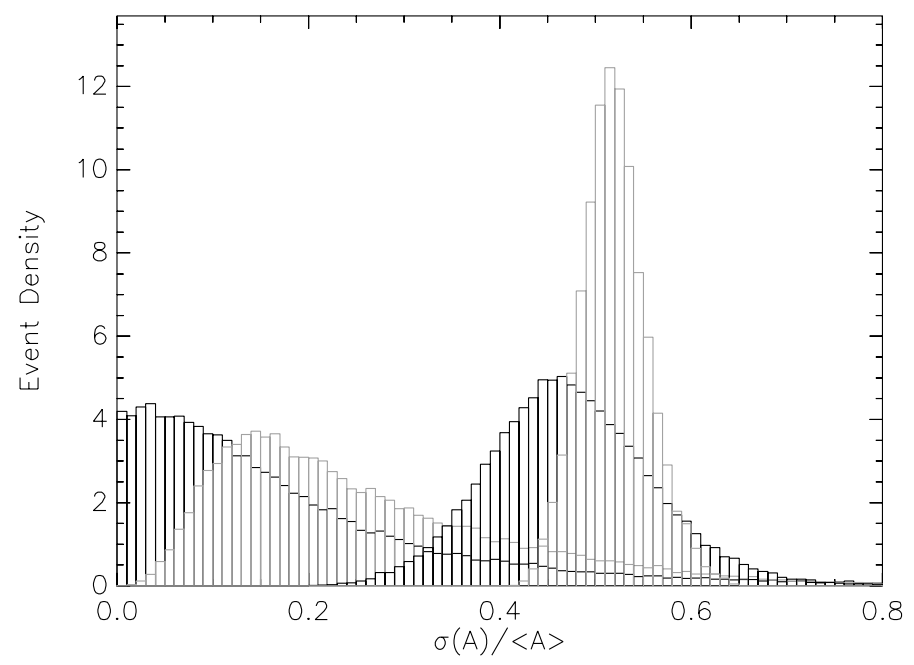

Fig. 3. Superposition of four histograms representing the $\sigma(A) /\langle A\rangle$ distribution. From left to right, the histograms correspond to sampling of 2, 5, 100 and 1000 amplitude values. The points were linearly sampled with separation 3 (explained in text). Each histogram comprises $2 \times 10^{4}$ points.

amplitude values. This, in turn, brings a sampling problem. To maximize the significance of the statistic we should pick amplitude values from time series that form an accurate sample of the amplitude distribution.

To address this question, we used the previously generated time series and amplitude values. Having generated a population of amplitude values, we studied this problem by picking ("sampling") amplitude values from the population and computing the sample $\sigma(A) /\langle A\rangle$. To determine what the sample $\sigma(A) /\langle A\rangle$ should be for different samplings, our procedure was to: (a) choose a sampling method and pick $n$ values of $A$ from our population; (b) compute the sample $\sigma(A) /\langle A\rangle$ and record it; (c) repeat the procedure many times $\left(10^{4}\right.$ to $\left.5 \times 10^{4}\right)$ and compute the histogram with all the values of $\sigma(A) /\langle A\rangle$. This allows us to construct the pdf of the sample $\sigma(A) /\langle A\rangle$, and compare it for different $n$ values and different samplings. It should be noted that this sample $\sigma(A) /\langle A\rangle$ distribution has a completely different nature from the amplitude distribution. This distribution is equivalent to the $\sigma(A) /\langle A\rangle$ distribution discussed in Pereira \& Lopes (2005).

After several "experiments", our results show that there are two important factors to consider in the sampling in order to get a correct estimate of the $\sigma(A) /\langle A\rangle$ value: the number of amplitude values $n$ and the type of sampling.

\subsection{Effect of the number of measurements}

The number of measurements of $A, n$, is the most important factor to consider when studying the significance of the statistic $\sigma(A) /\langle A\rangle$ (or, in other words, the distribution of the sample $\sigma(A) /\langle A\rangle)$. Figure 3 illustrates the effect of the number of measurements on the distribution of the sample $\sigma(A) /\langle A\rangle$. There are essentially two effects as we increase $n$ : (a) the type of the distribution starts by being almost exponential (for low values of $n$ ), and progressively acquires a Gaussian-like shape, and its peak converges to 0.52 as $n$ increases; (b) the width of the distribution (when the "Gaussian" form is acquired) decreases exponentially with $\mathrm{n}$. This can be seen in the figure, when $n=1000$ the full width at half maximum (FWHM) is somewhat narrower than $n=100$. 
In summary, the effect on the $\sigma(A) /\langle A\rangle$ distribution when increasing $n$ is a quick convergence of the modal bin/mean to 0.52 and an exponential diminution of the width. This effect can be seen in Fig. 4.

\subsection{Effect of the sampling type}

The number of measurements is the most important factor for correctly estimating $\sigma(A) /\langle A\rangle$. However, the statistic is also influenced by other factors, such as the type of sampling.

It is easier to explain the types of sampling by making a parallel between our sampling of amplitude values from the population and the observational reality. Let us suppose that each time series' segment where the amplitudes were determined corresponds to $6 \mathrm{~h}$ of continuous observation. Let us also suppose that in a telescope a given star was observed for $6 \mathrm{~h}$ every night, in $n$ consecutive nights. Given that on $24 \mathrm{~h}$ there are 4 "segments" of $6 \mathrm{~h}$ and that during one only could the star be observed, this means that for all the possible amplitude values $A_{i}, A_{i+1}, A_{i+2}$, $A_{i+3}, A_{i+4}, \ldots, A_{K}$, only the $A_{i}, A_{i+4}, \ldots, A_{i+4(n-1)}$ were observed. In terms of our amplitude population, where each value was determined in contiguous time series, this is equivalent to picking the $A_{i}, A_{i+4}, \ldots, A_{i+4(n-1)}$ values from the population (starting at given index $i$ ). We call this type of sampling linear sampling with separation 4 (because of the "jumps" in 4 between each value).

Linear sampling is the most realistic sampling type. Its effect on the significance of $\sigma(A) /\langle A\rangle$ was studied by experimenting with different separations. For the sake of completeness, uniform random sampling was also tested.

In the previous section it was noted that the effect of the number of measurements $n$ on the distribution of the sample $\sigma(A) /\langle A\rangle$ was two-fold: by increasing $n$ the distribution's modal bin converges (rapidly) to 0.52 , and its width converges (slowly) to zero. This convergence to the "true result" is defined, in distribution terms, as the convergence of the distribution of the sample $\sigma(A) /\langle A\rangle$ to a Dirac Delta distribution centered in 0.52. One can define a certain "speed of convergence" that specifies how quickly the distribution of the sample $\sigma(A) /\langle A\rangle$ converges to the true result. The effect of the sampling type on the distribution seems to be to slow down or increase these speeds of convergence. A "better" sampling (i.e., measurements are well-spaced in time) will result in a faster convergence of the modal bin to 0.52 and of the width to zero.

With linear sampling, it was found that as one increases the separation, the distribution generally seems to converge faster to the true result, as one increases $n$. This holds true up to some limiting speed of convergence. This result is not surprising, given that, by increasing the separation, we are extracting amplitude values that correspond to measurements with a larger temporal separation between them - increasing the statistical significance of the amplitude values. In addition, our testing of the uniform random sampling tells us that this is in fact the best sampling possible, defining the maximum speed of convergence - which again is not surprising, because it affords the best statistical significance to the amplitude values.

The effects of the number of measurements and the sampling type have been quantified in Fig. 4, where the morphology of the sample $\sigma(A) /\langle A\rangle$ is analyzed by looking at its modal bin and FWHM. The values of these two parameters of the distribution are plotted in the top and bottom panels as a function of $n$ and of the sampling type. It can be seen that the random uniform sampling sets the best theoretical limits on the sample $\sigma(A) /\langle A\rangle$ distribution, where the modal bin converges faster to the 0.52 value
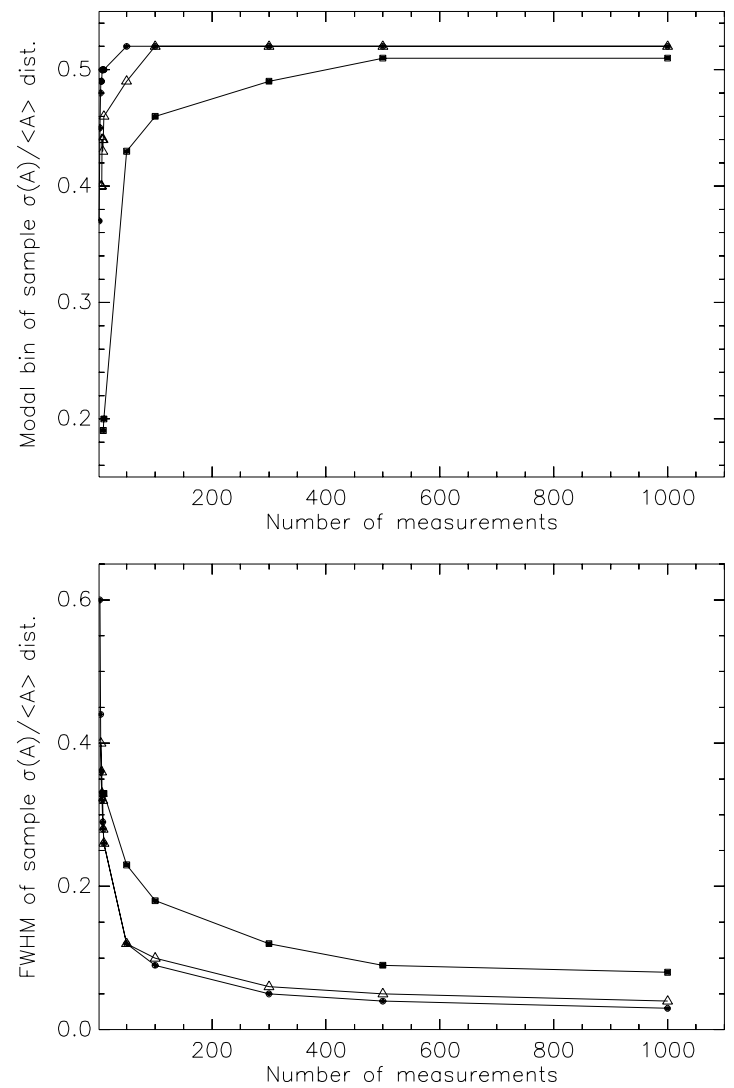

Fig. 4. Effect of the sampling type and number of measurements on the sample $\sigma(A) /\langle A\rangle$ distribution. Two parameters of the distribution are represented: modal bin (top) and FWHM (bottom). Symbols represent different types of sampling: linear sampling with separation 4 (squares); linear sampling with separation 24 (triangles) and uniform random sampling (circles).

and the width to zero. In terms of the linear sampling, it is clear that there is a faster convergence as we increase the separation.

One not-so-positive aspect (to observers) that is reflected in Fig. 4 is the high number of measurements needed to get a good estimate of $\sigma(A) /\langle A\rangle$. For instance, using linear sampling with separation 24 only with 50 measurements is the expected $\sigma(A) /\langle A\rangle$ distribution to have a modal bin around 0.49 with a width of 0.12 , which is acceptable for providing an accurate answer. But the number of possible amplitude measurements depends on the time scale of the oscillations. To accurately determine one amplitude value, a reasonable demand is that we observe three periods (in fact, it can be less than that). If we are considering oscillations like the solar 5 min oscillations, then in one night $(6-7 \mathrm{~h})$ we can have around 25 sets of three periods, or 25 measurements of amplitudes. On the other end of the scale, we have the $\gamma$ Doradus stars, with periods of several hours or even days that require multiple nights of observation to determine one amplitude value, so the aspect of the number of measurements when dealing with $\gamma$ Doradus stars is a clear disadvantage.

\section{Application to $\gamma$ Dor stars}

\subsection{Results for ground-based observations}

As seen in Fig. 4, our results show that one needs around 100 measurements to get an accurate measurement of $\sigma(A) /\langle A\rangle$. While this would not be a problem for stars with periods on the 


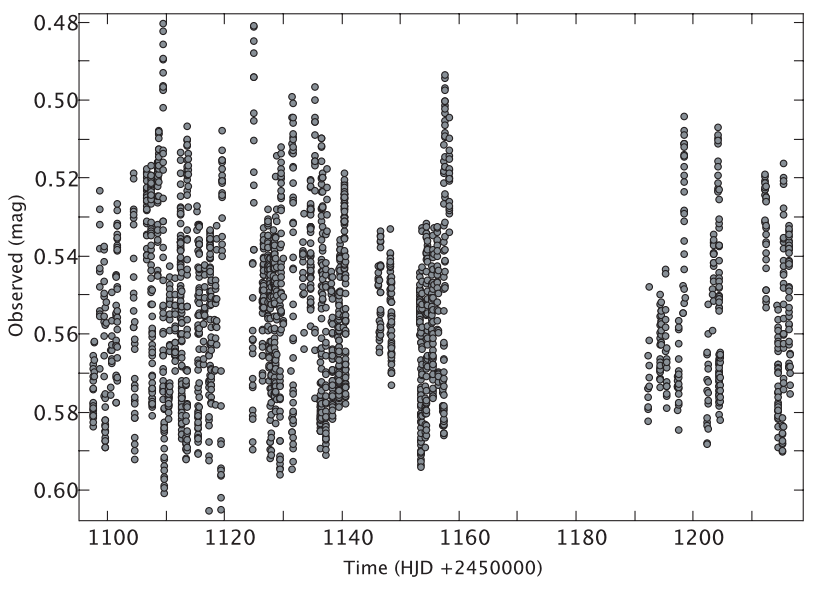

Fig. 5. Differential data set comprising multi-site observations of the $\gamma$ Doradus star HD 22702. Points corresponding to the $v$-band. Only a selected number of points were used in the analysis (see text).

order of minutes or hours, it is one in this case. For $\gamma$ Doradus stars, oscillation periods on the order of days means that one would need very large time series, with at least 100 nights of observations. This is a very ambitious target, even with large observational campaigns.

Despite the difficulties a priori, we have analyzed observational data from a $\gamma$ Doradus star and tried the method on these data - to see how far the method could be pushed with ground-based observations. The chosen star was HD 22702, a main-sequence star with $V=8^{\mathrm{m}} 81$ and spectral type A2 that belongs to the Pleiades open cluster (29 in Trumpler 1921). The observational data were taken from a photometric multisite campaign, from October 1998 to February 1999, in which the $\gamma$ Doradus nature of HD 22702 is confirmed (by SMR), using the $90-\mathrm{cm}$ telescope at Sierra Nevada Observatory (Granada, Spain), together with the 1.5-m telescope at San Pedro Mártir Observatory (Mexico). The data, shown in Fig. 5, include 53 useful nights, with a total span of 119 days. To get the amplitude measurements from the data, a compromise solution between quality/number was to extract from the original time series 11 amplitude measurements (each extracted in a set with 3 or 4 consecutive nights).

Using standard sine-fitting techniques with the Period 04 (Lenz \& Breger 2005) software package, the light curve for the Strömgren $v$ band was analyzed, analyzing three periods in each data subset. For each period, the statistics $\sigma(A)$ and $\langle A\rangle$ were computed over the 11 data sets. The results of the analysis could not be conclusive - the error bars we obtained for $\sigma(A) /\langle A\rangle$ were approximately $100 \%$ of the value. This result is likely to stem from the fact that we only have 11 measurements of amplitude (and, according to the simulations, $10^{2}$ measurements are needed for a theoretical error bar of 0.1 in $\sigma(A) /\langle A\rangle$ ). However, it was not obvious from the start (with a high quality time series) that one would only be able to get 11 measurements of amplitude. It might be possible to squeeze the data and get a few more, but there is always a tradeoff between the quality of each measurement (i.e., a measurement spanning many periods) and the number of measurements. But in the end, the result would not be very different: with this type of ground-based it is very difficult to apply the statistical method to detecting a signature of stochastic excitation in $\gamma$ Doradus stars.

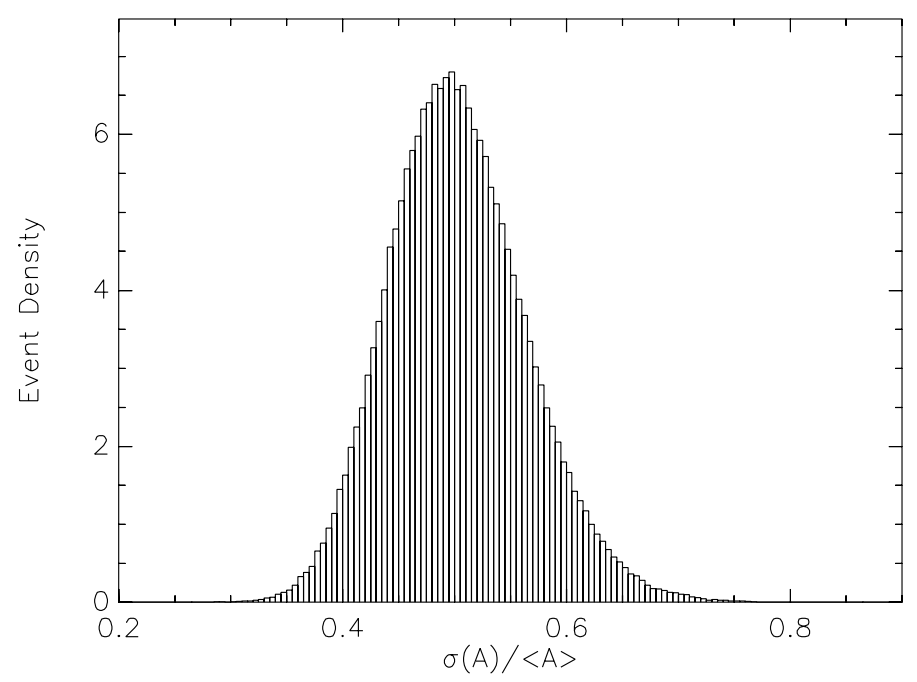

Fig. 6. Normalized histogram for the sample $\sigma(A) /\langle A\rangle$ distribution, with 71 measurements of amplitude, linearly sampled with separation 1 (equiv. to 6 months of observations with COROT).

\subsection{Predictions for the COROT space mission}

While probing the excitation mechanism in $\gamma$ Doradus stars via this proposed method is very difficult with ground-based observations, satellite observations may shed some light on this issue. With the advent of $\gamma$ Doradus stars in the field of view of the COROT space mission, six months of continuous data will be available for stars of this type. With this in mind, we look at how clearly defined the sample $\sigma(A) /\langle A\rangle$ would be for space observations, assuming stochastic excitation.

Based on the expected total time of observation with COROT, we can predict the distribution of the sample $\sigma(A) /\langle A\rangle$ for those observations. We assume that the observable $\gamma$ Doradus stars will have periods comparable to HD 22702, the $\gamma$ Doradus we analyzed previously. First, the approximate number of amplitude measurements is computed. In the HD 22702 case, the major mode has a period of approx. $2.57^{\mathrm{d}}$, meaning that there are approx. 71 periods in 6 months - yielding 71 amplitude values (if one value is determined per period). This was thus the number of amplitude values employed in our simulation (described in Sect. 4), with a run for $n=71$ and using linear sampling with separation 1 (contiguous values). The results of this run are plotted in Fig. 6.

For the equivalent of 6 months of COROT data, the expected $\sigma(A) /\langle A\rangle$ (modal bin) is around 0.5 with a FWHM of approximately 0.135 . We can see the distribution in Fig. 6, where it is evident that, while not perfect, a precise measurement of $\sigma(A) /\langle A\rangle$ can be made in the case of stochastic excitation. This means that, if stochastic excitation is present in the $\gamma$ Doradus star to be analyzed, one will be able to find the signature of stochastic excitation.

\section{Conclusions}

By analyzing the temporal variation of a mode's amplitude we can extract some information about the excitation mechanism. In this work we proposed to better determine the applicability conditions of the method used in Pereira \& Lopes (2005) and to look for its possible application to $\gamma$ Doradus stars.

Important information can be gathered from our simulations about the observational requirements of the method (given in number of amplitude measurements and spacing between 
measurements), and also about the analysis of closely-spaced modes. The simulations show that a considerable number of measurements (on the order of $10^{2}$ ) is needed to obtain a good error bar on the sample $\sigma(A) /\langle A\rangle$. Our numerical results also show that in the case of stochastic excitation, the amplitude distribution has the same properties when one measures the amplitude of a group of closely-spaced (unresolvable) modes. While this is very important in the case of $\gamma$ Doradus stars, both results are generally valid for any type of star.

An effort was made to test the method with observational data (ground-based observations) of a $\gamma$ Doradus star. However, the limited number of amplitude measurements we were able to gather did not allow for a conclusive result. According to the simulations a lot more measurements are necessary for a precise determination of $\sigma(A) /\langle A\rangle$. In the case of $\gamma$ Doradus stars this is a hard thing to achieve, since their periods are very long. We then turned our attention to the COROT space mission and the data it will produce. For the star in our case study, HD 22702, we show that the number of amplitude measurements determined with the time span of COROT are sufficient for one to make a good measurement of $\sigma(A) /\langle A\rangle$ and to look for a stochastic excitation signature.

\footnotetext{
Acknowledgements. This work was supported by a grant from Fundação para a Ciência e Tecnologia (reference number POCTI/FNU/50210/2003). T.M.D.P. acknowledges financial support also from Fundação para a Ciência e Tecnologia, reference number SFRH/BD/18726/2004. J.C.S. and S.M.R. acknowledge financial support from the European Marie Curie action MERG-CT-2004-513610. J.C.S. acknowledges support at the Instituto de Astrofísica de Andalucía by an I3P contract financed by the European Social Fund. PJA is supported by the program "Ramón y Cajal" of the Spanish "Ministerio de Educación y Ciencia". The Spanish CICYT (Comisión Interministerial de Ciencia Y Tecnología) is acknowledged for providing funds for the project with the reference number ESP2004-03855-C03-01.
}

\section{References}

Aerts, C., \& Kaye, A. B. 2001, ApJ, 553, 814

Aerts, C., \& Krisciunas, K. 1996, MNRAS, 278, 877

Aerts, C., Eyer, L., \& Kestens, E. 1998, A\&A, 337, 790

Arentoft, T., Bouzid, M. Y., Sterken, C., Freyhammer, L. M., \& Frandsen, S. 2005, PASP, 117, 601

Balona, L. A., Bohm, T., Foing, B. H., et al. 1996, MNRAS, 281, 1315

Choo, K. J., Kim, S.-L., Yoon, T. S., et al. 2003, A\&A, 399, 99

Dupret, M.-A., Grigahcène, A., Garrido, R., Gabriel, M., \& Scuflaire, R. 2004, A\&A, 414, L17

Dupret, M.-A., Grigahcène, A., Garrido, R., Gabriel, M., \& Scuflaire, R. 2005, A\&A, 435, 927

ESA 1997, VizieR Online Data Catalog, 1239, 0

Eyer, L., \& Aerts, C. 2000, A\&A, 361, 201

Gabriel, M. 1996, Bull. Astron. Soc. Ind., 24, 233

Grigahcène, A., Dupret, M.-A., Gabriel, M., Garrido, R., \& Scuflaire, R. 2005, A\&A, 434, 1055

Guzik, J. A., Kaye, A. B., Bradley, P. A., Cox, A. N., \& Neuforge, C. 2000, ApJ, 542, L57

Handler, G. 1999, MNRAS, 309, L19

Henry, G. W., \& Fekel, F. C. 2003, AJ, 126, 3058

Henry, G. W., Fekel, F. C., \& Henry, S. M. 2005, AJ, 129, 2815

Kaye, A. B., Handler, G., Krisciunas, K., Poretti, E., \& Zerbi, F. M. 1999, PASP, 111,840

Kim, S.-L., Chun, M.-Y., Park, B.-G., et al. 2001, A\&A, 371, 571

Kumar, P., Franklin, J., \& Goldreich, P. 1988, ApJ, 328, 879

Lenz, P., \& Breger, M. 2005, Commun. Asteroseismol., 146, 53

Martín, S. 2003, in Interplay of Periodic, Cyclic and Stochastic Variability in Selected Areas of the H-R Diagram, ASP Conf. Ser., 292, 59

Martín, S., \& Rodríguez, E. 2000, A\&A, 358, 287

Martín, S., Bossi, M., \& Zerbi, F. M. 2003, A\&A, 401, 1077

Moya, A., Suárez, J. C., Amado, P. J., Martín-Ruiz, S., \& Garrido, R. 2005, A\&A, 432, 189

Pereira, T. M. D., \& Lopes, I. P. 2005, ApJ, 622, 1068

Suárez, J. C., Moya, A., Martín-Ruiz, S., Amado, P. J., \& Garrido, R. 2005, A\&A, 443, 271

Trumpler, R. J. 1921, Lick Obs. Bull., 10, 110

Warner, P. B., Kaye, A. B., \& Guzik, J. A. 2003, ApJ, 593, 1049

Zerbi, F. M., \& Kaye, A. B. 2002, in Radial and Nonradial Pulsationsn as Probes of Stellar Physics, ASP Conf. Ser., 259, IAU Colloq., 185, 494

Zerbi, F. M., Rodríguez, E., Garrido, R., et al. 1999, MNRAS, 303, 275 